\title{
2 Ätiologie der Adipositas
}

\author{
Alfred Wirth
}

In Anbetracht der Adipositasepidemie in Deutschland ist die Frage nach der Entstehung dieser Krankheit berechtigt. Wenngleich hinsichtlich des Missverhältnisses von Energieaufnahme zu Energieverbrauch wissenschaftlich viele gesicherte Erkenntnisse vorliegen, tut sich der klinisch Tätige in der Regel schwer, im Einzelfall die Ursache der Adipositas zu eruieren.

Bei den meisten Menschen wird das Gewicht relativ konstant reguliert; größere Tagesschwankungen sind auf Verschiebungen im Wasserhaushalt zurückzuführen. Langfristig steigt das Gewicht im Alter zwischen 20 und 60 Jahren in Industrienationen um 15 bis $20 \mathrm{~kg}$; im höheren Alter geht es oft aus bisher unbekannten Gründen wieder zurück. Eine Gewichtszunahme im Laufe des Erwachsenenalters ist zwar normal, jedoch nicht physiologisch; bei Naturvölkern beträgt sie nur ca. $2 \mathrm{~kg}$.

\subsection{Erhöhte Energieaufnahme}

Komplexe Regulation von Hunger und Sättigung: Die Energieaufnahme ist äußerst komplex geregelt. Die Schaltstellen der Energieaufnahme sind, wie unten gezeigt wird, durch viele parallele Strukturen auf verschiedenen Ebenen und in zahlreichen Organen vernetzt (Arch 2002; s. Abb. 3). Die Regulation des Körpergewichtes ist jedoch keine reine Autoregulation durch biologische Mechanismen. Selbstverständlich kontrollieren Menschen ihr Körpergewicht auch kognitiv (bewusst).
Die Hunger-Sättigung-Regulation ist ein Beispiel par excellence für ein sog. biopsychosoziales Interaktionsmodell. Biologische Parameter im Gehirn, im Fettgewebe, im Intestinaltrakt und im Blut induzieren eine Wahrnehmung dafür, ob Hunger eintritt, gegessen oder das Essen beendet wird: Letztlich entscheiden jedoch übergeordnete zentralnervöse Strukturen über das Essverhalten.

Mit Aufnahme der Speisen in den Magen-DarmTrakt kommt es durch die Volumenzunahme zu einer Dehnung dieser Organe, was nervale und chemische Reize (Produktion oder Hemmung von Hormonen) zur Folge hat. Mit Beginn der Nährstoffresorption gelangen Metabolite (z.B. Clukose) über die Leber in andere Organe.

Im Hypothalamus befinden sich eine Reihe von biochemischen Substanzen sowie deren Rezeptoren, die eine Rolle als Neurotransmitter oder Neurohormone ausüben. Es handelt sich um Neurotransmitter wie Noradrenalin und Serotonin (5-Hydroxy-Tryptamin), aber um auch solche, die aus dem Gastrointestinaltrakt (z.B. Chrelin, Cholezystokinin) und dem Fettgewebe (z.B. Leptin) kommen.

Alimentäre Adipositas: Nicht nur Erwachsene, auch Kinder unterschätzen ihre Nahrungsaufnahme, je jünger desto ausgeprägter. Erwachsene und Kinder unter 8 Jahren geben im Mittel 40\% zu wenig Nahrungsmengen an. Es bestehen daher grundsätzliche Zweifel an den Angaben der Betroffenen, insbesondere zur Essmenge in einem Ernährungsprotokoll. 


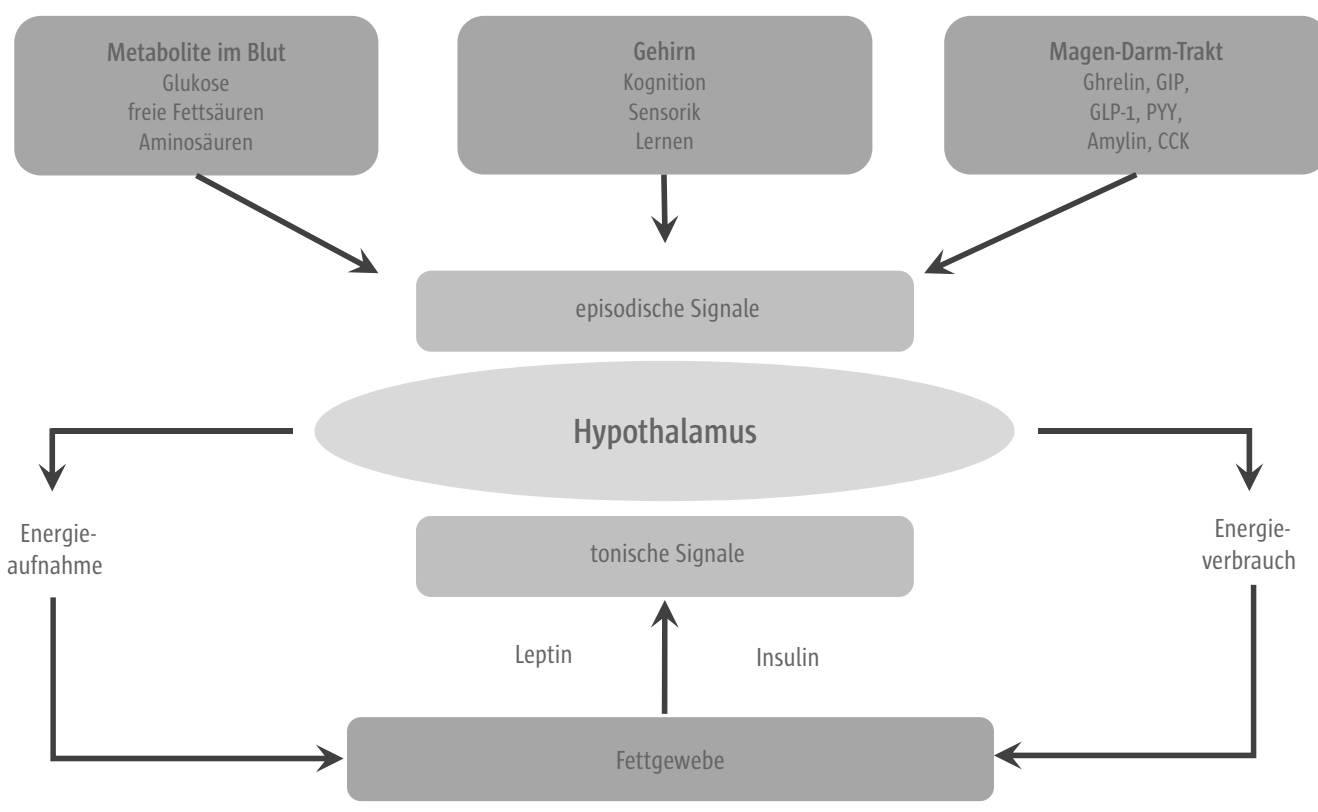

Abb. 3 Beschreibung der Regulation von Energieaufnahme und Energieverbrauch. Der Hypothalamus spielt dabei eine zentrale Rolle, da in ihm Signale von verschiedenen Körperregionen verarbeitet werden. (Wirth 2008, mit freundlicher Genehmigung von Springer Science and Business Media)

\section{Für eine alimentäre Adipositas gibt es 2 Gründe: Die Betroffenen essen entweder das Falsche oder sie es- sen zu viel.}

Die Bedeutung der Makronährstoffe: Fett in der Nahrung fördert die Gewichtszunahme, Fett enthält doppelt so viel Energie wie Kohlenhydrate oder Eiweiß. Adipöse essen mehr Fett als Normalgewichtige. Folgende Fragen drängen sich auf: Wird mehr Fett nicht nur aus Gründen der Schmackhaftigkeit, sondern auch wegen mangelnder Sättigung konsumiert? Verhalten sich Adipöse anders als Normalgewichtige? Viele Untersuchungen zu diesem Thema zeigen, dass Fett wenig sättigt und Mahlzeiten mit hohem Fettgehalt und damit großem Energiegehalt keine verstärkte oder länger anhaltende Sättigung bewirken. Da fettreiche Speisen gut schmecken und daher oft in übergroßer Menge gegessen werden, spricht man von „passive overconsumption“.

Fette Speisen verleiten zu hoher Energieaufnahme durch:

- Schmackhaftigkeit

- hohe Energiedichte

- geringes Volumen

- geringe Sättigung
Zwischen komplexen Kohlenhydraten (z.B. Vollkornbrot) und Zucker besteht hinsichtlich der Nahrungsaufnahme ein großer Unterschied. Kaum untersucht sind zuckerhaltige Speisen wie Süßspeisen, Speiseeis, Kuchen, Kekse usw. Relativ gute Daten gibt es inzwischen jedoch zu zuckerhaltigen Getränken, zu Softdrinks und Säften. Kinder, die Wasser trinken, haben ein niedrigeres Gewicht. In den USA hat sich der Konsum von Softdrinks in den letzten 50 Jahren verfünffacht; bei uns ist die Entwicklung möglicherweise ähnlich. Da inzwischen der Anteil des Zuckers in Getränken den Hauptanteil am Zuckerkonsum darstellt, sind Getränke für die Gewichtsentwicklung sehr wichtig (Ludwig et al. 2001).

\section{Vor allem Zucker in Getränken erhöht das Gewicht.}

Der Konsum an Süßstoffen (Saccharin, Cyclamat, Aspartam oder Acesulfam-K) ist weit verbreitet, um Zucker geschmacklich - ohne deren Energiegehalt $\mathrm{zu}$ imitieren. In den 8oer-Jahren berichtete eine Arbeitsgruppe über vermehrten Hunger unter Aspartam; in den meisten nachfolgenden Studien wurde dies nicht bestätigt. Süßstoffe werden daher auch 


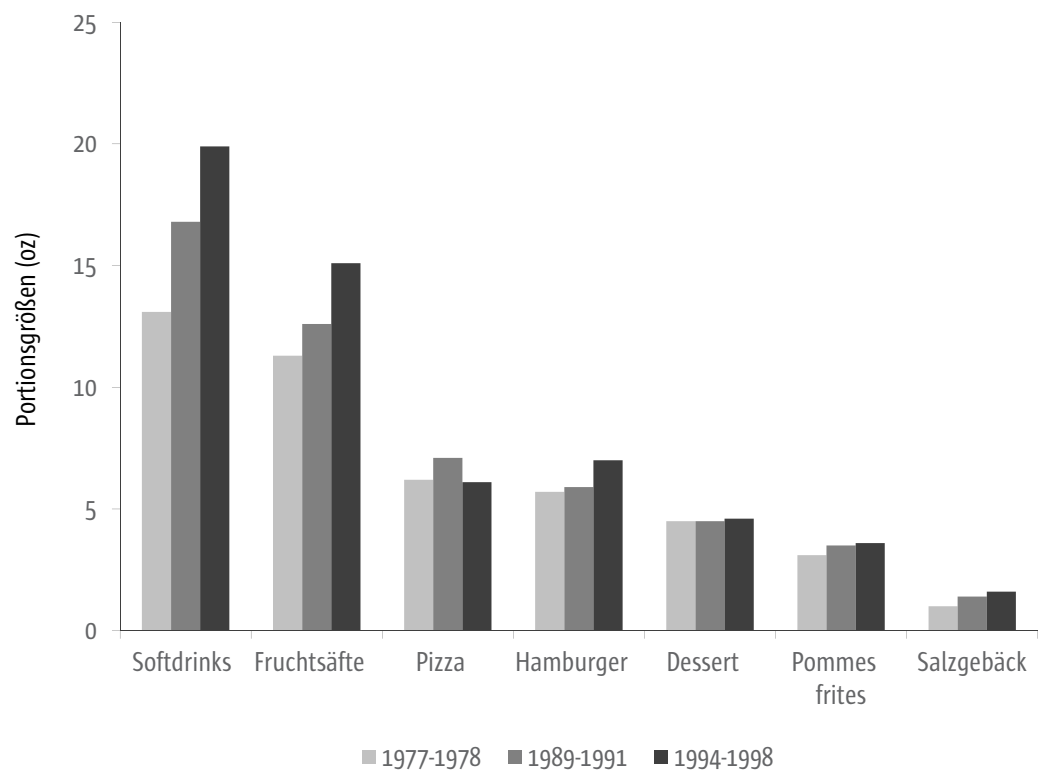

Abb. 4 Zunahme der Portionsgrößen verschiedener Nahrungsmittel in den USA von 1977-1998 (nach Nielsen et al. 2003)

von Fachgesellschaften in Leitlinien zur Gewichtsabnahme empfohlen.

Die Portionsgrößen werden häufig übersehen: Viele Menschen nehmen wenig Fett und Zucker zu sich und werden dennoch übergewichtig. Ursache hierfür sind u.a. zunehmende Packungsgrößen in den letzten Jahren. Aus den USA liegen Beobachtungen über 20 Jahre vor, wonach vor allem die Packungsgrößen für Softdrinks und Fruchtsäfte etwa um die Hälfte zunahmen (Nielsen et al. 2003; s. Abb. 4). Auch bei uns muss man feststellen, dass 1,5- und 2-Liter-Packungen früher nicht oder kaum angeboten wurden. Das Gleiche trifft auf Snacks, Pommes frites, Hamburger und Cheeseburger zu. Wenn die Packungs- und Portionsgrößen zugenommen haben, ist noch nicht gesichert, dass auch größere Mengen konsumiert werden. Experimentelle Studien zeigen aber, dass große Packungen und große Portionen auch zu vermehrter Energieaufnahme führen. Wer viel auf dem Teller hat, isst offensichtlich auch viel. Auch schnelles Essen und Essen bis zur vollen Sättigung erhöhen das Gewicht (Maruyama et al. 2008).

Häufigkeit von Mahlzeiten - Essfrequenz: Wer häufig isst, ist auch dicker? Die meisten Untersuchungen, auch Metaanalysen, fanden keinen Zusammenhang zwischen der Häufigkeit der Mahlzeiten und der konsumierten Nahrungsenergie und dem Body-Mass-
Index (BMI). Sicher ist nur, dass man keine Zwischenmahlzeiten einhalten soll, es sei denn, man hat Hunger. Wer jedoch abends größere Mahlzeiten zu sich nimmt, muss möglicherweise mit einer $\mathrm{Ce}$ wichtszunahme rechnen. Am Abend führen Kohlenhydrate/Zucker zu einer stärkeren Insulinsekretion; auch andere Hormone sind aktiver, sodass die Bildung von Körperfett begünstigt ist.

Fastfood: In der CARDIA-Study nahm in einer 15jährigen Beobachtung der Fastfoodkonsum zu (Pereira et al. 2005). Wer schon zu Beginn der Untersuchung > 2-mal/Woche Fastfood aß und in der Beobachtungszeit auf eine dritte Fastfoodmahlzeit überging, nahm durchschnittlich $16 \mathrm{~kg}$ an Gewicht zu. Vorwiegend jüngere Personen verzehrten häufig Fastfood; sie hatten eine hohe Energieaufnahme mit viel Fett und raffinierten Zuckern sowie Softdrinks und wenig Ballaststoffen.

Fastfood zeichnet sich durch große Portionen, Schmackhaftigkeit, hohe Energiedichte und einen hohen glykämischen Index aus.

Glykämischer Index: Steigen Blutzucker und damit auch Insulin nach einer Mahlzeit wenig an, hat man Nahrungsmittel mit einem niedrigen glykämischen In- 
dex zu sich genommen. Die Datenbasis für die Annahme, dass Lebensmittel mit niedrigem glykämischen Index das Körpergewicht verringern, ist zwar schwach, aber dennoch gegeben. Empfehlenswert sind daher z.B. Vollkornbrot statt Weißmehlbrötchen und Möhren statt Bananen.

Energiedichte: Erst in den letzten Jahren wurde deutlich, dass die Energiedichte von Nahrungsmitteln, d.h. der Quotient aus Energiegehalt (Kalorien) und Menge (g), für die Entwicklung der Adipositas von großer Bedeutung ist (Schusdziarra et al. 2008). Wer Nahrungsmittel mit hoher Energiedichte zu sich nimmt - diese sind meist stark fett- und zuckerhaltig - hat wenig auf dem Teller, aber dennoch viele Kalorien zu sich genommen. Wer hingegen z.B. Corned beef statt Leberwurst und Harzer Käse statt Emmentaler isst, steht mit der Energiebilanz günstiger da.

\subsection{Verminderter Energieverbrauch}

Zur Genese der Adipositas trägt ein geringer Energieverbrauch ebenso bei wie eine erhöhte Energieaufnahme. Der Energieverbrauch des Menschen setzt sich aus 3 Komponenten zusammen:

- dem Grundumsatz,

- der Thermogenese (Wärmebildung) und

- der körperlichen Aktivität.

Grundumsatz: Der Anteil des Grundumsatzes (GU) am Gesamtenergieverbrauch beträgt beim Erwachsenen $55-70 \%$. Er wird im Wesentlichen vom Alter, Geschlecht, der fettfreien Körpermasse (Muskelmasse) und genetischen Voraussetzungen bestimmt. In einer gut kontrollierten Untersuchung konnten Ravussin und Mitarbeiter (1988; s. Abb. 5) zeigen, dass eine Gewichtszunahme von $10 \mathrm{~kg}$ innerhalb von 4 Jahren bei Probanden mit einem niedrigen Grundumsatz 8-mal häufiger vorkam als bei solchen mit einem hohen. Für klinische Zwecke kann der Grundumsatz nicht aus Tabellen abgelesen, sondern nur direkt gemessen werden, da er individuell stark schwankt.

Körperliche Inaktivität: Während Crundumsatz und Thermogenese nur wenig beeinflussbar sind, sind bei der körperlichen Aktivität naturgemäß große Variationen möglich. Man unterscheidet eine spontane und eine fakultative (willentliche) Aktivität, letztere lässt sich beeinflussen. In einer großen europäischen Multicenterstudie mit 15.239 Teilnehmern konnte überzeugend nachgewiesen werden, dass sowohl körperliche Inaktivität (Sitzen) als auch geringe Aktivität das Adipositasrisiko deutlich erhöhen (Martinez-Gonzales et al. 1999; s. Abb. 6).

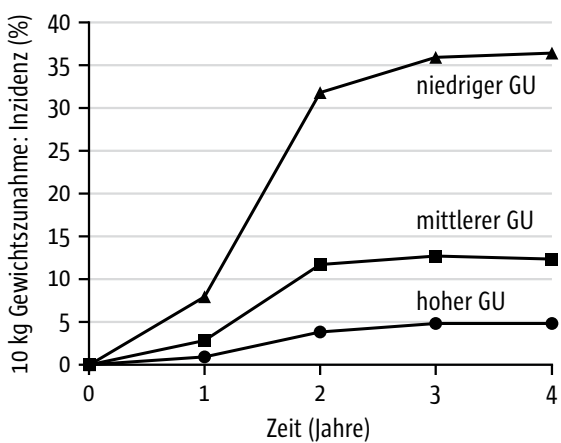

Abb. 5 Auswirkungen eines niedrigen, mittleren und hohen Grundumsatzes auf die Gewichtsentwicklung innerhalb von 4 Jahren (nach Ravussin et al. 1988)

Wer viel sitzt und sich wenig bewegt, hat ein um das 4-fache erhöhtes Risiko, adipös zu werden.

\subsection{Genetische Prädisposition}

Adipositas kann vererbt sein; die Wahrscheinlichkeit hierfür liegt bei 50-60\%. Mutationen in Genen sind selten, üblicherweise besteht jedoch ein Polymorphismus. In Familien-, Adoptions- und Zwillingsstudien hat man das Ausmaß genetischer Faktoren an der Entwicklung der Adipositas erforscht. Die Vererbung spielt vorwiegend beim Energieverbrauch eine Rolle. Weniger Erkenntnisse gibt es über die Energieaufnahme, wenngleich die neuere Forschung auch hierfür Hinweise auf eine genetische Determination liefert.

Wesentliche Erkenntnisse stammen aus der Zwillingsforschung. Zwillinge wurden meist unter- oder übernährt. Auch Familienstudien liefern Hinweise für die Rolle der Vererbung bei der Entwicklung der Adipositas. Wenn die Varianz von Körpergewicht oder viszeraler Fettmasse innerhalb von Familien (oder Zwillingspaaren) deutlicher variiert als die Varianz zwischen den Familien (oder Zwillingspaaren), weist der Unterschied auf eine erbliche Komponente hin (Stunkard et al. 1986).

\section{Die Adipositas wird zu etwa 50\% vererbt.}

In Adoptionsstudien kann man genetische Effekte erforschen, da die Adoptierten mit ihren biologischen Eltern die Erbmasse und mit den Adoptiveltern die 


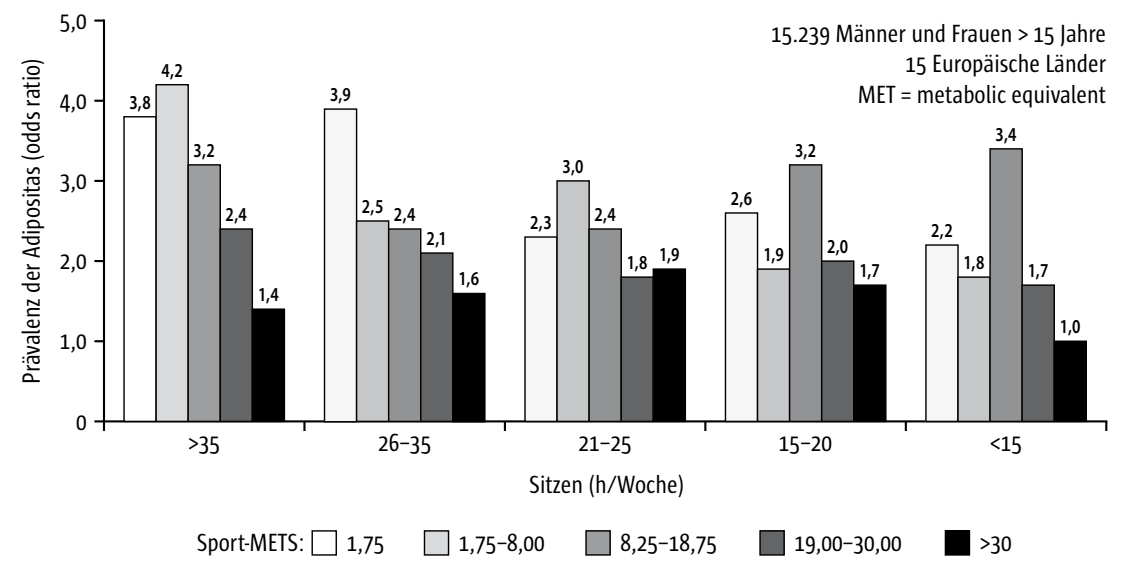

Abb. 6 Entwicklung der Adipositas bei körperlich Aktiven und Inaktiven in 15 Ländern Europas. Die körperliche Aktivität ist in MET („metabolic equivalent“) angegeben. 1 MET entspricht dem Energieverbrauch in Ruhe. (nach Martinez-Gonzalez et al. 1999)

Umwelt teilen. Eine Auswertung des dänischen Adoptionsregisters mit 3.580 Personen war diesbezüglich sehr aufschlussreich (Stunkard et al. 1986): Das Gewicht der Adoptierten korrelierte nicht mit dem der Adoptiveltern, sondern nur mit dem der biologischen Eltern.

Ein wichtiger Ansatz ist die Suche nach Kandidatengenen. Am bekanntesten wurde eine Mutation im Leptin-Cen bei Cousin und Cousine blutsverwandter Eltern einer pakistanischen Familie. Ihre Leptinspiegel waren extrem niedrig; das Mädchen wog im Alter von 8 Jahren bereits $86 \mathrm{~kg}$ bei einer Körperlänge von $137 \mathrm{~cm}$.

Eine Reihe von genetischen Syndromen mit Adipositas sind beschrieben. Sie sind selten und haben nicht nur Veränderungen der Fettmasse und der Fettverteilung zur Folge, sondern auch andere Anomalien (z.B. Prader-Willi-Syndrom).

\subsection{Schlafmangel - gestörter Schlaf}

In den letzten Jahren gab es eine Reihe von Hinweisen, dass Umfang und Qualität des Schlafes einen Einfluss auf das Körpergewicht haben. Immer mehr Menschen verlagern ihre Aktivitäten in die späten Abendstunden und die Nacht. Viele haben inzwischen die Hauptmahlzeit von der Mittagszeit in die Abendstunden verlegt. Schon vor Jahrzehnten trug das Fernsehen dazu bei, die Abendstunden auszudehnen und den Beginn des Nachtschlafs zu verschieben. Inzwischen sind viele Menschen abends am Computer tätig, nicht nur um privat im Internet zu surfen, sondern auch um berufliche Arbeit vor- und nachzu- arbeiten. Die Bewältigung großer Distanzen mittels Flugzeug geht mit Jetlags einher. Ähnlich ist die Situation bei Schichtarbeitern, die inzwischen einen Anteil von $>20 \%$ in unserer Gesellschaft ausmachen .

Computertätigkeit und Fernsehen am Abend verschieben die Bettgehzeit nach hinten und die Schichtarbeit sowie Jetlags führen durch eine Störung des Biorhythmus häufig zu Schlafdefiziten. Nachgewiesen ist von all diesen Charakteristika unserer technisierten Welt, dass sie die Entstehung von Adipositas begünstigen (Garaulet et al. 2010). Im vergangenen Jahrhundert hat die Schlafdauer um 1,5 Stunden abgenommen, ein Drittel der Erwachsenen schläft weniger als 6 Stunden.

Bei der Untersuchung von 1.024 30-60 Jahre alten Personen in den USA mittels Polysomnografie fand man einen U-förmigen Zusammenhang zwischen Schlafdauer und dem BMI (Taheri et al. 2004; s. Abb. 7). Personen mit einer Schlafdauer von 7,8 Stunden hatten den niedrigsten BMI. Wer weniger als diese Zeitspanne pro Nacht schlief - das waren immerhin $74 \%$ der Untersuchten - wies einen deutlich höheren BMI auf. Ähnlich war die Situation bei 6.862 Kindern im Alter von 5-6 Jahren in Bayern (von Kries et al. 2002). $2,1 \%$ der Kinder mit einem Nachtschlaf $>11,5$ Stunden waren adipös; schliefen sie $<10$ Stunden, betrug die Adipositashäufigkeit 5,4\%.

Der Zusammenhang zwischen Schlafdauer und Körpergewicht bzw. Körperfettmasse gemessen mit der bioelektrischen Impedanzanalyse (BIA) bestand unabhängig von anderen Risikofaktoren für die Adipositas. 


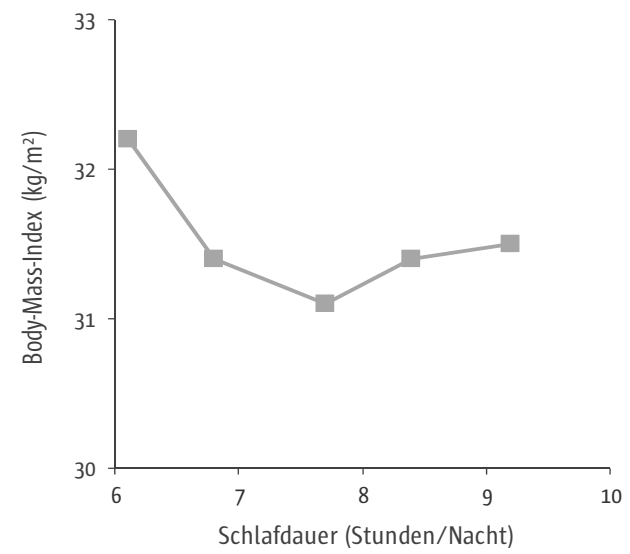

Abb. 7 Zusammenhang zwischen Schlafdauer und BodyMass-Index bei 1.024 US-Amerikanern im Alter von 30 bis 60 Jahren (Taheri et al. 2004)

Was bewirkt eine Gewichtszunahme bei Schlafmangel? Die Gründe für das Phänomen Adipositas und Schlafmangel sind bisher nur ansatzweise erforscht. Bekannt ist, dass wenig Schlaf mit verstärktem Hunger einhergeht. Verkürzt man den Schlaf experimentell auf unter 4 Stunden über 2 Nächte, so nimmt der Hunger um 24\% zu (van Gauter et al. 2008).

\subsection{Pharmaka können Gewichtszunahmen verursachen}

Antidepressiva: Vorwiegend trizyklische und heterozyklische Antidepressiva führen durch Stimulation von Hunger und Appetit zur Gewichtszunahme Pharmaka mit zusätzlicher anticholinerger Wirkung bewirken eine Mundtrockenheit und induzieren so eine vermehrte Flüssigkeitsaufnahme, was in der Regel auch eine erhöhte Energieaufnahme bedeutet. Himmerich et al. (2005) haben Substanzen der verschiedenen Wirkungsklassen auf Basis der vorhandenen Literatur ihren gewichtssteigernden Effekten entsprechend gruppiert (s. Tab. 3).

Neuroleptika/Antidepressiva: Durch die Blockierung von Dopaminrezeptoren und Beeinflussung weiterer Rezeptoren kann es zur Gewichtszunahme von 3-4 kg innerhalb eines Vierteljahres kommen.

Insulin und orale Antidiabetika: Insulin, ein Wachstumshormon, erhöht über verschiedene Mechanismen das Körpergewicht um 5 bis $10 \mathrm{~kg}$ (Wirth u. Hauner 2010). Auch Sulfonylharnstoffe und Glinide bedingen eine Gewichtszunahme.
Tab. 3 Gewichtszunahme unter psychopharmakologischer Behandlung (Himmerich et al. 2005)

\begin{tabular}{|c|c|c|c|}
\hline & hoch & mäßig & gering \\
\hline $\begin{array}{l}\text { Anti- } \\
\text { depressiva }\end{array}$ & $\begin{array}{l}\text { Amitriptylin } \\
\text { Doxepin } \\
\text { Maprotilin } \\
\text { Mirtazapin } \\
\text { Trimipramin }\end{array}$ & $\begin{array}{l}\text { Clomipramin } \\
\text { Imipramin } \\
\text { Nortriptylin }\end{array}$ & $\begin{array}{l}\text { Citalopram } \\
\text { Fluoxetin } \\
\text { Flavoxamin } \\
\text { Moclobemid } \\
\text { Sertralin } \\
\text { Tranylcypromin }\end{array}$ \\
\hline $\begin{array}{l}\text { Phasen- } \\
\text { prophylak- } \\
\text { tika }\end{array}$ & $\begin{array}{l}\text { Lithium } \\
\text { Valproat }\end{array}$ & Carbamazepin & $\begin{array}{c}\text { Gabapentin } \\
\text { Lamotrigin } \\
\text { Topiramat }\end{array}$ \\
\hline $\begin{array}{l}\text { Anti- } \\
\text { psychotika }\end{array}$ & $\begin{array}{c}\text { Clozapin } \\
\text { Olanzapin }\end{array}$ & $\begin{array}{c}\text { Zuclopenthixol } \\
\text { Quetiapin } \\
\text { Risperidon }\end{array}$ & $\begin{array}{c}\text { Amisulprid } \\
\text { Aripiprazol } \\
\text { Haloperidol } \\
\text { Ziprasidon }\end{array}$ \\
\hline
\end{tabular}

Kortikosteroide: Kortisol erhöht das Körpergewicht, typischerweise in Form einer abdominalen Adipositas mit rundlichem Gesicht, Striae rubrae, Muskelschwäche und Osteoporose. Kortisol stimuliert den Appetit und die Fettneubildung.

Östrogene/Kontrazeptiva: Östrogene im Rahmen einer Hormonersatztherapie erhöhen das Körpergewicht nicht, Kontrazeptiva hingegen begünstigen durch eine Hemmung der Fettoxidation die Körperfettvermehrung.

$\beta$-Blocker: $\beta$-Blocker führen zu einer leichten Gewichtszunahme. Sie senken den Sympathikotonus und damit den Energieverbrauch und hemmen die Lipolyse sowie die Fettoxidation.

\section{Zusammenfassung}

- Die Vererbung der Anlage „Adipositas“ hat einen Anteil von etwa $50 \%$.

- Ein niedriger Ruheenergieumsatz führt langfristig zur Gewichtszunahme. Es handelt sich um ein genetisches Phänomen.

- Die Adipositas kann sich auch in Form eines genetischen Syndroms manifestieren; diese Syndrome sollte man differenzialdiagnostisch berücksichtigen.

- $\quad$ An der Regulation von Hunger und Sättigung sind verschiedene neurohumorale Substanzen beteiligt.

- Der Sättigungseffekt von Makronährstoffen ist wie folgt: Eiweiß > Kohlenhydrate > Fett.

- Energiedichte Lebensmittel (Fett, Zucker oder beides) führen aufgrund einer hohen Schmackhaftigkeit und oft geringen Sättigung zur Gewichtszunahme. 
- Unser Lebensstil mit Bewegungsmangel und geringer beruflicher Muskelarbeit erklärt vor allem die zunehmende Adipositashäufigkeit in den letzten Jahrzehnten.

- Adipöse bewegen sich oft weniger als Normalgewichtige.

- Auch Medikamente können zur Gewichtszunahme führen.

\section{Literatur}

Arch JRS (2002) Lessons in obesity from transgenic animals. J Endocrinol Invest 25, 867-875

van Cauter E, Knutson KL (2008) Sleep and the epidemic of obesity in children and adults. Eur | Endocrinol 159, S. 59-66

Garaulet M, Ordovás JM, Madrid JA (2010) The chronobiology, etiology and pathophysiology of obesity. Int J Obes 34, 1667-1683

Himmerich H, Schuld A, Pollmächer T (2005) Gewichtszunahme unter Psychopharmakotherapie. Dtsch Arztebl 102, C1735C1740

Ludwig DS, Petersen KE, Gortmaker SL (2001) Relation between consumption of sugar-sweetened drinks and childhood obesity: a prospective, observational study. Lancet 357, 505-508

Martinez-González MA, Martinez JA, Hu FB, Gibney MJ, Kearney J (1999) Physical inactivity, sedentary lifestyle and obesity in the European Union. Int | Obes 23, 1192-1201

Maruyama K, Sato S, Ohira T, Maeda K, Noda H, Kubota Y, Nishimura S, Kitamura A, Kiyama M, Okada T, Imano H, Nakamura M,
Ishikawa Y, Kurokawa M, Sasaki S, Iso H (2008) The joint impact of being overweight of self reported behaviours of eating quickly and eating until full: cross sectional survey. BMJ 337, a1926a1931

Nielsen S), Popkin BM (2003) Patterns and trends in food portion sizes, 1977-1998. JAMA 289, 450-453

Pereira MA, Kartashov Al, Ebbeling CB, van Horn L, Slattery ML, Jacobs Jr DR, Ludwig DS (2005) Fast-food habits, weight gain, and insulin resistance (the CARDIA study): 15-year prospective analysis. Lancet 365, 36-42

Ravussin E, Lillioja S, Knowler WC, Christin L, Freymond D, Abbott WG, Boyce V, Howard BV, Bogardus C (1988) Reduced rate of energy expenditure as a risk factor for body-weight gain. $\mathrm{N}$ Engl I Med 318, 467-472

Schusdziarra V, Hausmann M, Kröner E (2008) Satt essen und abnehmen. Individuelle Ernährungsumstellung ohne Diät. MMI-Verlag

Stunkard AJ, Sørensen TI, Hanis C, Teasdale TW, Chakraborty R, Schull WJ, Schulsinger F (1986) An adoption study of human obesity. N Engl I Med 314, 193-198

Taheri S, Lin L, Austin D, Young T, Mignot E (2004) Short sleep duration is associated with reduced leptin, elevated ghrelin, and increased body mass index. Plos Medicine 1, 210-217

Wirth A, Engeli S, Hinney A, Reinehr T (2008) Adipositas: Ätiologie, Epidemiologie, Folgekrankheiten, Diagnostik, Therapie. Springer-Verlag, Stuttgart, 3. Auflage

Wirth A, Hauner H (Hrsg.) (2010) Das Metabolische Syndrom. Urban \& Vogel, München 2. aktualisierte Auflage

\section{(2) \\ Prof. Dr. med. Alfred Wirth \\ Klinische Ausbildung in Innerer Medizin, Kardiologie, Sportmedizin, Ernährungsmedizin. Von 1977-1978 Stipendiat der Deutschen Forschungsgemeinschaft an der Universität in Göteborg, 1983 Habilitation, 1993 apl. Professor. 1983-2009 Ärztlicher Direktor der Klinik Teutoburger Wald in Bad Rothenfelde/Niedersachsen, einer Schwerpunktklinik für Kardio- logie, Stoffwechsel (Diabetes, Adipositas) und Pneumologie. Seit 1992 Leiter der Fort- und Weiterbildungsveranstaltung für Sportärzte auf Langeoog. Mitglied in diversen Fachge- sellschaften (z.B. DAG, DGK), über 200 Publikationen in nationalen und internationalen Zeitschriften.}

